\title{
Komplizierte Malaria tropica: spezifische und supportive Therapie bei importierten Erkrankungen
}

\author{
F. v. Sonnenburg, Th. Löscher, H. D. Nothdurft und L. Prüfer \\ Medizinische Klinik Innenstadt (Direktor: Prof. Dr. E. Buchborn), Abteilung für Infektions- und Tropenmedizin (Leiter: Prof. Dr. W. Lang), der Universität München
}

Bei elf von 43 nichtimmunen Patienten mit Malaria tropica traten eine oder mehrere Organkomplikationen auf: zerebrale Malaria, akute respiratorische Insuffizienz, akutes Nierenversagen, Sekundärinfektion, Autoimmunhämolyse, spontane Milzruptur und akute Pankreatitis. Die Parasitämie betrug 0,1 bis $60 \%$. Initiale antiparasitäre Therapie mit Chinin parenteral führte in neun Fällen zu rascher Rückbildung der Parasitämie. Zusätzlich wurde ein zweites schizontozides Mittel entsprechend der Resistenzlage gegeben. Die supportive Therapie umfaßte intensivmedizinische Überwachung mit Bilanzierung von Elektrolyt- und Wasserhaushalt sowie gegebenenfalls eine frühzeitige Hämodialyse und (oder) endotracheale Intubation mit PEEP-Beatmung. In einem Fall mit exzessiver Parasitämie wurde eine Austauschtransfusion durchgeführt. Heparin wurde nur bei nachgewiesener disseminierter intravasaler Gerinnung gegeben, Corticosteroide nur bei persistierender Autoimmunhämolyse. Alle Patienten überlebten ohne zurückbleibende Defekte. Die retrospektive Analyse zeigt, daß neben einer raschen spezifischen Therapie die supportive Behandlung der einzelnen Organkomplikationen für Verlauf und Prognose der komplizierten Malaria tropica mitentscheidend ist.
Complicated falciparum malaria: specific and supportive therapy for imported diseases

Eleven of 43 nonimmune patients with falciparum malaria had one or several organ complications: cerebral malaria, acute respiratory failure, acute renal failure, secondary infection, autoimmune haemolysis, spontaneous spleen rupture, and acute pancreatitis. Parasitaemia was 0.1 to $60 \%$. Initial antiparasitic therapy with quinine given parenterally resulted in rapid regression of parasitaemia. An additional schizonticide agent was given depending on parasitic resistance. Supportive therapy comprised intensivecare monitoring including fluid and electrolyte balance and, if necessary, early haemodialysis and (or) endotracheal intubation with PEEP breathing. In one patient with excessive parasitaemia exchange transfusion was performed. Heparin was given only in proven disseminated intravascular coagulation, corticosteroids only in persistent autoimmune haemolysis. All patients survived without suffering permanent defects. Retrospective analysis shows that, apart from rapid specific therapy, supportive treatment of the individual organ complications determines course and prognosis of complicated falciparum malaria.
Aus Statistiken des Bundesgesundheitsamtes ist in den letzten Jahren eine deutliche Zunahme eingeschleppter Malaria tropica abzulesen. Das ist einerseits auf den noch immer steigenden internationalen Reiseverkehr in Malaria-Endemiegebiete zurückzuführen, andererseits entwickelt sich in einigen Gebieten zunehmend eine Resistenz gegen alle Medikamente, die bisher in der Malariaprophylaxe angewandt wurden. Nichtimmune, die in gemäßigten Klimazonen aufgewachsen sind und nicht schon von Geburt an den Infektionen ausgesetzt waren, sind besonders gefährdet (9). Die Letalität der Malaria tropica bei Tropenrückkehrern liegt nach Angaben des Bundesgesundheitsamtes bei 9,7\%. Meist sterben Patienten an den nicht mehr beherrschbaren Organkomplikationen.

Dtsch. med. Wschr. 111 (1986), 934-938

(C) 1986 Georg Thieme Verlag Stuttgart · New York
Um Hinweise auf den Stellenwert verschiedener supportiver Maßnahmen bei Organkomplikationen zu erhalten, haben wir retrospektiv die klinischen Daten und supportiven Maßnahmen bei elf Patienten mit komplizierter Malaria tropica analysiert.

\section{Patienten und Symptomatik}

Von 1983 bis Dezember 1985 wurden in unserer Abteilung 43 Patienten mit akuter Malaria tropica behandelt.

Bei elf Patienten traten Komplikationen auf (Tabelle 1). Der relativ hohe Anteil an Komplikationen erklärt sich daraus, daß uns einige Patienten wegen des schweren Verlaufes zugewiesen wurden.

Tabelle 2 gibt die klinische Symptomatik sowie die Therapie bei den elf Patienten wieder.

Sieben Patienten wiesen eine pulmonale Beteiligung mit mehr oder weniger ausgeprägter respiratorischer Insuffizienz auf. Die röntgenologischen Veränderungen entsprachen immer einem interstitiellen Lungenödem. 
Tab. 1. Angaben über elf Tropenreisende mit komplizierter Malaria tropica

\begin{tabular}{|c|c|c|c|c|}
\hline Fall & $\begin{array}{c}\text { Alter } \\
\text { (Jahre), } \\
\text { Geschlecht }\end{array}$ & Aufenthalisort & $\begin{array}{l}\text { Prophy- } \\
\text { laxe* }\end{array}$ & $\begin{array}{c}\text { Parasit- } \\
\text { ämie }\end{array}$ \\
\hline 1 & $22 \delta$ & Äthiopien & keine & $35 \%$ \\
\hline 2 & $64 \delta$ & Kenia & keine & $60 \%$ \\
\hline 3 & $63 q$ & Kenia/Tansania & $\mathrm{CQ}$ & $35 \%$ \\
\hline 4 & $44 \%$ & Kenia & keine & $20 \%$ \\
\hline 5 & 460 & Philippinen & keine & $25 \%$ \\
\hline 6 & 430 & Sierra Leone & keine & $10 \%$ \\
\hline 7 & 430 & Kenia/Tansania & $\begin{array}{c}F \text { (unregel- } \\
\text { mäßig) }\end{array}$ & $15 \%$ \\
\hline 8 & 390 & Zentral-/Ostafrika & $\mathrm{CQ}$ & $2 \%$ \\
\hline 9 & $15 \delta$ & Kenia & $\mathrm{CQ}$ & $0,1 \%$ \\
\hline 10 & $38 \approx$ & Kenia & $\mathrm{CQ}$ und $\mathrm{F}$ & $1 \%$ \\
\hline 11 & $43 q$ & Tansania & $\mathrm{CQ}$ & $15 \%$ \\
\hline
\end{tabular}

${ }^{*} \mathrm{CQ}=$ Chloroquin $\left(\right.$ Resochin $\left.{ }^{\circledR}\right)$

$\mathrm{F} \quad=$ Pyrimethamin/Sulfadoxin (Fansidar $\left.{ }^{\circledR}\right)$
Alle Patienten wurden intensivmedizinisch betreut, und dementsprechend wurde eine genaue Bilanzierung des Wasser- und Elektrolythaushaltes durchgeführt. Zentrale Venendruckmessungen schlossen eine Überwässerung aus. Bei drei Patienten (Fall 1, 2 und 5) war Überdruckbeatmung (PEEP) notwendig.

Bei acht Patienten beobachteten wir eine zerebrale Beteiligung, die von Verwirrtheit und Somnolenz bis zum tiefen Koma reichte. Ein Schädel-Computertomogramm wurde nur bei einem Patienten (Fall 5) angefertigt; Hinweise auf ein Hirnödem fanden sich hierbei nicht. Bemerkenswert ist bei diesem Patienten, daß sich trotz sofortigen Therapiebeginns auf der Aufnahmestation innerhalb einer Stunde aus völlig klarer Bewußtseinslage ein komatöser Zustand entwickelte.

Der Grad der Hämolyse war häufig größer, als nach dem Prozentsatz der parasitierten Erythrozyten zu erwarten gewesen wäre. Lediglich bei einem Patienten (Fall 8) setzte sich auch nach Elimination der Parasitämie die Hämolyse in beträchtlichem Ausmaß fort. Der Patient wies einen positiven direkten Coombs-Test mit positi-

Tab. 2. Klinik und Therapie bei elf Patienten mit komplizierter Malaria tropica

\begin{tabular}{|c|c|c|c|c|c|c|}
\hline Fall & Komplikationen & $\begin{array}{l}\min . \\
\text { Hämoglobin } \\
(\mathrm{g} / \mathrm{d} !)\end{array}$ & $\begin{array}{l}\text { min. Throm- } \\
\text { bozytenzah } \\
\left(\times 10^{3}\right)\end{array}$ & $\begin{array}{l}\text { Kreatinin } \\
\text { (Harnstoff-N) } \\
(\mathrm{mg} / \mathrm{d})\end{array}$ & spezifisch & $\begin{array}{l}\text { Therapie* } \\
\text { zusätzlich }\end{array}$ \\
\hline 1 & $\begin{array}{l}\text { zerebral (Koma Grad III), } \\
\text { puimonal (Lungenödem), } \\
\text { renal (eingeschränkte Nierenfunktion) }\end{array}$ & 8,1 & 16 & 2,8 & $\mathrm{C} / \mathrm{CQ}$ & Beatmung (PEEP) \\
\hline 2 & $\begin{array}{l}\text { zerebral (Koma Grad III-IV, Krämpfe), } \\
\text { pulmonal (Lungenödem), } \\
\text { renal (oligurisches Nierenversagen) }\end{array}$ & 8,6 & 21 & $\begin{array}{c}5,6 \\
(179)\end{array}$ & $\mathrm{C} / \mathrm{T} / \mathrm{M}$ & $\begin{array}{l}\text { Austauschtransfusion } \\
\text { Beatmung (PEEP) } \\
\text { Hämodialyse }\end{array}$ \\
\hline 3 & $\begin{array}{l}\text { pulmonal (respiratorische Insuffizienz), } \\
\text { Begleitpankreatitis }\end{array}$ & 6,8 & 14 & 1,2 & $\mathrm{C} / \mathrm{F} / \mathrm{M}$ & $\begin{array}{l}\mathrm{O}_{2} / \mathrm{CPAP} \\
\text { parenterale Ernährung }\end{array}$ \\
\hline 4 & $\begin{array}{l}\text { zerebral (Somnolenz) } \\
\text { pulmonal (respiratorische Insuffizienz) }\end{array}$ & 8,1 & 10 & 1,2 & $\mathrm{C} / \mathrm{M}$ & $\mathrm{O}_{2} / \mathrm{CPAP}$ \\
\hline 5 & $\begin{array}{l}\text { zerebral (Koma Grad III, Krämpfe), } \\
\text { pulmonal (Lungenödem), } \\
\text { renal (oligurisches Nierenversagen), } \\
\text { Sekundärinfektion (Tuberkulose) }\end{array}$ & 6,6 & 12 & $\begin{array}{l}4,1 \\
(85)\end{array}$ & $\mathrm{C} / \mathrm{M}$ & $\begin{array}{l}\text { Hämodialyse } \\
\text { Beatmung (PEEP) } \\
\text { Tuberkulostase }\end{array}$ \\
\hline 6 & $\begin{array}{l}\text { zerebral (Somnolenz) } \\
\text { pulmonal (respiratorische Insuffizienz), } \\
\text { renal (eingeschränkte Nierenfunktion) }\end{array}$ & 9,7 & 44 & 2,0 & $\mathrm{C} / \mathrm{R}$ & $\mathrm{O}_{2} / \mathrm{CPAP}$ \\
\hline 7 & $\begin{array}{l}\text { zerebral (Somnolenz), } \\
\text { renal (polyurisches Nierenversagen), } \\
\text { Milzruptur }\end{array}$ & 6,9 & 11 & $\begin{array}{l}4,7 \\
(84)\end{array}$ & $\mathrm{C} / \mathrm{M}$ & Splenektomie \\
\hline 8 & $\begin{array}{l}\text { Coombs-positive Hämolyse, } \\
\text { tiefe Beinvenenthrombose }\end{array}$ & 5,3 & 98 & 0,9 & $F$ & $\begin{array}{l}\text { Steroide } \\
\text { Heparin }\end{array}$ \\
\hline 9 & $\begin{array}{l}\text { Sekundärinfektion (Verdacht auf } \\
\text { Listeriose-Meningoenzephalitis) }\end{array}$ & 11,8 & 53 & 1,3 & $\mathrm{C} / \mathrm{F}$ & Penicillin \\
\hline 10 & $\begin{array}{l}\text { zerebral (Somnolenz), } \\
\text { pulmonal (respiratorische Insuffizienz), } \\
\text { disseminierte intravasale Gerinnung }\end{array}$ & 10,5 & 8 & $\begin{array}{l}2,5 \\
(39)\end{array}$ & $\mathrm{C} / \mathrm{M}$ & $\begin{array}{l}\mathrm{O}_{2} / \mathrm{CPAP} \\
\text { Heparin }\end{array}$ \\
\hline 11 & zerebral (Koma Grad III) & 8,5 & 12 & 0,9 & $\mathrm{C} / \mathrm{M}$ & Intubation \\
\hline $\begin{array}{l}\text { PEEF } \\
\text { CPA } \\
\text { C } \\
\text { CQ }\end{array}$ & \multicolumn{6}{|c|}{$\begin{array}{ll}=\text { positiv endexpiratorischer Druck } & \mathrm{F} \\
=\text { kontinuierlich positiver Atemwegsdruck } & \mathrm{M} \\
=\text { Chinin } & \mathrm{T} \\
=\text { Chloroquin (Resochin }^{\circledR} \text { ) } & \end{array}$} \\
\hline
\end{tabular}


vem C3d auf. Außer gewaschenen Erythrozyten wurden Steroide angewandt.

Häufig fand sich eine Nierenbeteiligung, eine längerdauernde Hämodialyse war jedoch nur bei zwei Patienten notwendig (Fall 2 und 5). Wegen einer Parasitämie von etwa 50-60\%, Koma Grad III-IV, Hämoglobinabfalls von 16 auf $7 \mathrm{~g} / \mathrm{dl}$ in den 8 Stunden vor Aufnahme bei uns und einer beatmungspflichtigen Ateminsuffizienz mit röntgenologischen Zeichen des Lungenödems schätzten wir die Prognose bei dem einen Patienten (Fall 2) als sehr schlecht ein. Wir entschlossen uns deshalb, neben den üblichen therapeutischen Maßnahmen einen sofortigen Blutaustausch unter Dialysebedingungen durchzuführen (10 Liter Vollblut in 12 Stunden).

Bei zwei Patienten (Fall 5 und 9) kam es während bzw. nach der Therapie der Malaria zu einer Zweitinfektion. Der eine Paticnt (Fall 9) war bei stationärer Einweisung nahezu parasitenfrei, eine Therapie war bereits in Kenia durchgeführt worden. Bei ihm bestand das Bild einer Meningoenzephalitis. Die humoralen und liquorständigen Antikörpertiter gegen Listeria monocytogenes stiegen innerhalb von 10 Tagen signifikant an; Penicillintherapie besserte die Symptomatik. Eine Listeriose scheint uns somit hinreichend wahrscheinlich, obwohl der direkte Erregernachweis nicht gelang. Erhebliche diagnostische Schwierigkeiten bereitete die während Beatmung aufgetretene offene Tuberkulose des anderen Patienten (Fall 5). Es bestand zunächst der Verdacht, daß er aspiriert haben könnte.

Ein Patient (Fall 7) erlitt ohne feststellbare mechanische Belastung - eine »tiefe Milzpalpation « wurde nicht durchgeführt - eine spontane Milzruptur. Die Blutung kam nicht zum Stillstand, so daß eine Splenektomie notwendig wurde.

Nur bei einem Patienten (Fall 10) konnte eine disseminierte intravasale Gerinnung mit Verbrauchskoagulopathie nachgewiesen werden; er wurde mit Heparin behandelt.

Bei einem Patienten (Fall 8) kam es während der Liegezeit zu einer tiefen Beinvenenthrombose. Eine Patientin (Fall 3) erkrankte an einer akuten Begleitpankreatitis ohne sonographisch nachweisbare Veränderungen im Gallenwegs- oder Pankreasbereich.

\section{Ergebnisse und Diskussion}

\section{Antiparasitäre Therapie}

In allen Fällen, in denen es um eine möglichst schnelle Reduktion der Parasitämie ging, behandelten wir mit Chinin. Bereits nach wenigen Stunden erzielten wir regelmäßig einen deutlichen Effekt. Wir sahen uns deshalb nicht veranlaßt, auf das Antiarrhythmikum Chinidin zurückzugreifen, obwohl es nach einzelnen Literaturberichten wirksamer sein soll (20).

Zur endgültigen Sanierung ist die Gabe eines zweiten schizontoziden Mittels empfehlenswert (8), da eine Chinin-Monotherapie nicht immer zur definitiven Aushei-
Tab. 3. Antiparasitäre Therapie der komplizierten Malaria tropica

Chinin- $\mathrm{HCl} 20-25 \mathrm{mg} / \mathrm{kg}$ pro 24 Stunden in 2-3 langsamen (über $1-2$ h) Infusionen (in NaCl $0,9 \%$ oder Glucose $5 \%$ ).

Bei Niereninsuffizienz oder ausgeprägter Leberbeteiligung muß die Dosis auf $10-15 \mathrm{mg} / \mathrm{kg}$ täglich reduziert werden.

Sobald wie möglich Umstellung auf orale Gabe. Dann ist zusätzlich ein zweites Schizontenmittel (Chloroquin, Pyrimethamin/Sulfadoxin. Mefloquin, eventuell Tetracyclin) entsprechend der Resistenzlage emptehlenswert

Therapiedauer bei Monotherapie und Kombination mit Tetracyclin 10 Tage, sonst bis zum Verschwinden der Parasiten.

lung führt. Die Wahl dieses Medikaments hängt weitgehend vom Herkunftsgebiet der eingeschleppten Malaria und der dort möglichen oder mutmaßlichen Resistenzlage ab (Tabelle 3).

Notwendig ist eine engmaschige Kontrolle der Parasitendichte, um Therapieversagen frühzeitig zu erkennen. Nach unseren Erfahrungen eignet sich der In-vitro-Resistenztest als Hilfestellung zur Therapie wenig. Standardisiert ist der Test bisher nur für Chloroquin, das Ergebnis ist erst nach 24-72 Stunden ablesbar, in etwa 25\% aller angesetzten Tests kommt es zu keinem Ergebnis. Der Invitro-Resistenztest unterscheidet nicht zwischen $\mathrm{R}_{\mathrm{l}^{-}}, \mathrm{R}_{\mathrm{II}^{-}}$ und $\mathrm{R}_{\mathrm{II}}$-Resistenzen und zeigt in etwa $25 \%$ zu häufig Resistenzen an (17).

\section{Pulmonale Beteiligung}

Während in älteren Arbeiten und Lehrbüchern eine pulmonale Beteiligung als Komplikation der Malaria tropica selten erwähnt wird, wird das Lungenödem zunehmend als einer der wesentlichen letalen Faktoren beschrieben $(4,9,10,15)$. Bei unseren Patienten war eine Ateminsuffizienz die zweithäufigste Komplikation, röntgenologisch fanden sich meist die Zeichen eines interstitiellen Lungenödems. Die Entwicklung eines Lungenödems wurde früher im wesentlichen auf Überwässerung zurückgeführt, da Patienten mit Malaria tropica oft zu Unrecht als exsikkiert eingeschätzt wurden. Bei unseren Patienten kam es zu keiner Überwässerung, so daß andere pathogenetische Faktoren vorliegen müssen. Vermutet werden kapillartoxische Substanzen, die ein der Schocklunge ähnliches Bild erzeugen $(6,10)$. Trotzdem sollte man den zentralen Venendruck überwachen, um eine Flüssigkeitsüberlastung zu verhïten. Ausreichende Gasaustauschbedingungen sollten nötigenfalls mit positivem endexspiratorischem Druck (PEEP) sichergestellt werden, um zu hohe inspiratorische Sauerstoffkonzentrationen zu vermeiden.

\section{Zerebrale Malaria}

Auch die Pathophysiologie der zerebralen Malaria ist noch nicht endgültig aufgeklärt. Bei Sektionsbefunden werden regelmäßig Hirnödeme und zerebrale Ringblutungen gefunden (9). Aus neueren computertomographischen Befunden (13) wird jedoch geschlossen, daß das 
Hirnödem erst in der agonalen Phase auftritt und deshalb nicht die Ursache für die zerebrale Malaria ist. Auch in dem Fall, in dem bei uns ein Schädelcomputertomogramm angefertigt wurde, fanden sich keine Hinweise auf ein Hirnödem trotz Streckkrämpfen und Koma-Grad III. Eine prospektive Studie bei 100 Patienten mit zerebraler Malaria hat keinen nützlichen Effekt von Steroiden nachweisen können (19). Die Bewußtlosigkeit hatte bei den mit Steroiden behandelten Patienten länger angehalten als bei der Vergleichsgruppe, Komplikationen traten häufiger auf. Allerdings wurden die Patienten dieser Studie nicht auf ein Hirnödem hin untersucht.

In jedem Fall ist eine zerebrale Beteiligung bei einer Malaria tropica prognostisch besonders ungünstig. Man sollte keinesfalls die Geschwindigkeit unterschätzen, mit der sich ein komatöser Zustand entwickeln kann. Deshalb sollte auch jede Malaria tropica bei Nichtimmunen stationär behandelt werden.

Obwohl wir uns dieser Problematik bewußt waren, trübte bei einem unserer Patienten (Fall 5) das Bewußtsein kurzfristig unbemerkt auf der Station ein. Das führte später insofern zu Komplikationen, als ein pulmonaler Herdbefund lange als Aspiration gedeutet wurde, ehe schließlich Mycobacterium tuberculosis nachgewiesen werden konnte.

Beeindruckend war bei einigen unserer Patienten, wie selbst tiefstes Koma folgenlos überwunden werden konnte. Das wird auch in der Literatur beschrieben (13, 20).

\section{Renale Komplikation}

Nierenbeteiligung bei Malaria tropica ist häufig. Selbst bei unkomplizierten Verläufen kommt es in nahezu $50 \%$ zu einer Proteinurie, die nicht mit der Höhe des Fiebers korreliert (7). Retrospektive Analysen bei Verstorbenen ergaben nahezu immer eine akute Niereninsuffizienz (9, 18). Die Pathogenese der akuten Niereninsuffizienz scheint multifaktoriell zu sein. Angenommen werden hämodynamische Veränderungen, Mikrozirkulationsstörungen, aber auch der massive Anfall von Hämolyseabbauprodukten, besonders von freiem Hämoglobin. Bei sich entwickelnder Niereninsuffizienz sollte rechtzeitig mit der Dialyse begonnen werden, um Hyperhydratation und Elektrolytverschiebungen zu vermeiden.

\section{Hämolyse}

Drei Mechanismen werden als Ursache für die Anämie bei der Malaria tropica angesehen: 1. Die Erythrozyten zerplatzen beim Freiwerden der Merozoiten, 2. es findet eine vermehrte Erythrozytenphagozytose statt, und 3. toxische Substanzen schädigen die Membran parasitierter und nicht parasitierter Erythrozyten. Zahlreiche Untersuchungen belegen, daß der Grad der Hämolyse meist größer ist, als nach dem Prozentsatz parasitierter Erythrozyten zu erwarten wäre. Meist ist jedoch die Hämolyse an das Vorhandensein von Parasiten gebun- den, und nach parasitologischer Heilung nimmt auch die Hämolyse $a b$ (1). In einzelnen Fällen verselbständigt sich jedoch die Hämolyse und setzt sich trotz geringer Parasitendichte oder nach völliger Elimination der Parasiten fort.

Die Ursache dieser verselbständigten Hämolyse wird in der Literatur kontrovers diskutiert $(2,3)$. Man nimmt an, daß es sich um einen autoimmunhämolytischen Prozeß handelt, obwohl der Coombs-Test selten positiv ist. Bei einem unserer Patienten (Fall 8) allerdings war der direkte Coombs-Test positiv, die Komplementkomponente C3d konnte nachgewiesen werden. Möglicherweise lag in diesem Fall eine medikamentenbedingte Immunhämolyse vom "Innocent-bystander «-Typ vor, da der Patient mit Chinin behandelt wurde. Bei autoimmunbedingter Hämolyse nach Malaria tropica wird Steroiden ein nützlicher Effekt zugeschrieben (2). Die normale Hämolyse, die während der Parasitämie auftritt, wird von Steroiden nicht beeinflußt (3).

Bei dramatischem Hämoglobinabfall kann die Gabe von Erythrozytenkonzentraten notwendig werden, obwohl die Anämie selten lebensbedrohlich ist $(9,10)$.

Ebenfalls kontrovers wird in der Literatur diskutiert, welchen Anteil bei schwerer Malaria tropica eine disseminierte intravasale Gerinnung hat $(10,12)$. Während bei allen unseren Patienten eine Thrombozytopenie auftrat, konnte eine disseminierte intravasale Gerinnung nur bei einem Patienten nachgewiesen werden. In diesem Fall entschlossen wir uns zur Heparintherapie. Inwieweit man eine niedrig dosierte Heparinprophylaxe durchführen sollte, bleibt wohl der individuellen Abwägung vorbehalten. Immerhin trat bei einem unserer Patienten eine tiefe Beinvenenthrombose auf.

Ein immunsuppressiver Effekt der Malaria ist seit langem bekannt und in Tierversuchen nachgewiesen (2). Deshalb sollte man immer auf Sekundärinfektionen achten, wie sie sich bei zwei unserer Patienten (Fall 5 und 9) zeigten.

Eine besondere kardiale Komplikation der Malaria tropica ist selten (9). Im Rahmen der Gesamterkrankung und der intensivtherapeutischen Maßnahmen wurden jedoch auch mehrere unserer Patienten katecholaminpflichtig.

Von Milzrupturen bei Malaria tropica wird immer wieder berichtet; daher sollte man eine allzu intensive Palpation der Milz vermeiden.

In einem besonders schweren Fall von Malaria tropica (Fall 2) hatten wir uns zum Blutaustausch entschlossen. Diese Therapieform ist noch im experimentellen Stadium. Es empfiehlt sich eine besonders sorgfältige Abwägung zwischen Nutzen und den nicht unerheblichen Risiken. Es bedarf spezieller Erfahrung mit dieser Technik, um die Vielzahl der Herz-Kreislauf-, Elektrolythaushaltund Kompartimentprobleme zu beherrschen. Hier sei nur in Stichworten der mögliche Nutzeffekt angeführt: Parasitierte und auch nicht parasitierte, aber vorgeschädigte Erythrozyten werden durch gesunde ersetzt, Ery- 
throzytenabbauprodukte, besonders freies Hämoglobin, werden ebenso wie mögliche Zytotoxine entfernt. In der Pathogenese verschiedener Organkomplikationen werden, wie schon erwähnt, sowohl Hämolyse und dadurch freiwerdende Erythrozytenabbauprodukte als auch zytotoxische Substanzen immer wieder als Ursache diskutiert. Das Überleben unseres Patienten ist kein ausreichender Beweis für die Wirksamkeit dieser Therapieform; allerdings sind in der Literatur in einzelnen Fällen ähnliche Erfolge beschrieben $(11,14,16,21)$.

\section{Literatur}

(1) Aikawa, M., M. Suzuki, Y. Gutierrez: Pathology of malaria. In Kreier, J. P. (Ed.): Malaria, Vol, 2 (Academic Press: New York 1980), 47.

(2) Bruce-Chwatt, L. J.: Essential Malariology (William Heinemann: London 1985)

(3) Charoenlarp, P., S. Vaniyanouta, P. Chat-Panyoporu: The effect of prednisone on red cell survival in patients with falciparum malaria. S. E. Asian J. trop. Med. publ. Hlth 10 (1979), 127.

(4) Deaton, J. G.: Fatal pulmonary edema as a complication of acute falciparum malaria. Amer. J. trop. Med. Hyg. 19 (1970), 196.

(5) Dietrich, M., W. Höfler, W. Lang, H. Wernsdorfer: Aktuelle Prophylaxe und Therapie der Malaria - Stand 1985. Dtsch. Ärztebl. (im Druck). ment with quinine and mefloquine or pyrimethamine-sulfadoxine for falciparum malaria. Brit. med. J. 1977/1, 1626.

(9) Horstmann, R. D., J. H. H. Ehrich, J. Beck, M. Dietrich: Letale Komplikationen der Malaria tropica bei Nichtimmunen. Dtsch. med. Wschr. 110 (1985) 1651 .

(10) James, M. F. M.: Pulmonary damage associated with falciparum malaria. A re- port of ten cases. Ann. trop. Med. Parasit. 79 (1985), 123

(11) Kramer, S. L., C. C. Campell, R. E. Moncrieff: Fulminant plasmodium falciparum infection treated with exchange blood transfusion. J. Amer. med. Ass. 249 (1983), 244

(12) Kurathong, S., T. Srichaikal, P. Isarangkura et al.: Exchange transfusion in cerebral malaria complicated by disseminated intravascular coagulation. S. E.

Asian J. trop. Med. publ. Hlth 10 (1979) 389

(13) Looareesuwan, S., N. J. White, D. A Warrel, P. Sutharasamai et al.: Do pa tients with cerebral malaria have cerebral oedema? Lancet 1983/I, 434.

(14) Neilsen, R. L., R. B. Kohler, W. Chin et al.: Case report. The use of exchange transfusions: a potentially useful adjunct in the treatment of fulminant falciparum malaria. Amer. J. med. Sci. 277 (1979), 325.

(15) Punyaguputa, S., T. Srichaikul, P. Nitiyanunt, B. Petchclai: Acute pulmonary insufficiency in falciparum malaria. Summary of 12 cases with evidence of disseminated intravascular coagulation. Amer. J. trop. Med. Hyg. 23 (1974), 557.

(16) Roncoroni, A. J., O. A. Martino: Therapeutic use of exchange transfusion in malaria. Amer. J. trop. Med. Hyg. 28 (1979), 440

(17) Smrkovski, L. L., R. L. Buck, A. K. Alcantara, C. S. Rodriguez, C. V. Uylang$\mathrm{co}$ : Studies of resistance to chloroquine, quinine, amodiaquine and mefloquine among Philippine strains of Plasmodium falciparum. Trans. roy. Soc. trop. Med. Hyg. 79 (1985), 37.

(18) Stone, W. J., J. E. Hanchett, J. H. Knepshield: Acute renal insufficiency due to falciparum malaria. Arch. intern. Med. 129 (1972), 620.

(19) Warrell, D. A., S. Looareesuwan, M. J. Warrell, P. Kasemsarn, R. Intaraprasert, D. Bunnag, T. Harinasuta: Dexamethasone proves deleterious in cerebral malaria. New Engl. J. Med. 306 (1982), 313.

(20) White, N. J., S. Looareesuwan, O. A Warell, T. Chongsuphajaisiddi, D. Bunnag, T. Harinasuta: Quinidine in falciparum malaria. Lancet 1981/1/, 1069. (21) Yarrish, R. L., J. S. Janas, J. S. Nosanchuk et al.: Transfusion malaria. Treatment with exchange transfusion after delayed diagnosis. Arch. intern. Med. 142 (1982), 187

Dr. F. v. Sonnenburg, Dr. Th. Löscher, Dr. H. D. Nothdurft, Dr. Luise Prüfer Abteilung für Infektions- und Tropenmedizin Medizinische Klinik Innenstadt der Universität 8000 München 40, Leopoldstr. 5 